\title{
Editorial: COVID Ecology and Evolution: Systemic Biosocial Dynamics
}

\author{
Matteo Convertino ${ }^{1 *}$ and Salvatore Flavio Pileggi ${ }^{2}$ \\ ${ }^{1}$ bluEco Lab, Tsinghua Shenzhen International Graduate School, Institute of Environment and Ecology, Tsinghua University, \\ Shenzhen, China, ${ }^{2}$ Centre on Persuasive Systems for Wise Adaptive Living (PERSWADE), Faculty of Engineering \& IT, \\ University of Technology Sydney, Sydney, NSW, Australia
}

Keywords: biocomplexity, environmental epidemiology, phylogenetics, healthcare, ecosystems, environmental change, pollution, complex networks

\section{Editorial on the Research Topic}

\section{COVID Ecology and Evolution: Systemic Biosocial Dynamics}

In a vastly impacted world by COVID-19, it is widely observable how ecosystem heterogeneity-i.e., biological and environmental more importantly, where the latter include socio-economical aspects-plays a fundamental role in shaping COVID-19 space-time impact and response (Figure 1). The lack to address ecosystem heterogeneity, that is partially reflected by administrative boundaries, within the decision making process can cause cascading extreme risks with multiple effects beyond morbidity and mortality in the human population. This is observed in many areas implementing disorganized control strategies or assuming non science-based "one size fits all" approaches. An ecosystem approach is needed where the collective ecology of COVID-19 is taken into account, from the portfolio of its determinants to controls. During the COVID-19 pandemic, due to a reduced human activity (e.g., in industrial production and mobility), surprising positive environmental outcomes also occurred, such as the decrease of air and water pollution. Meanwhile, many other still open research questions arose, such as the spread of SARS-CoV-2 through the hydrologic cycle and pathways.

In light of the above considerations this Special Issue aimed to embrace a systemic analysis of COVID-19 considering the multifaceted reality of the problem, including basic research and practical implications. Thirteen papers were published accordingly, spanning biology (Cueno and Imai; Cueno et al.), environmental epidemiology (Pansini and Fornacca; Rahalkar and Bahulikar; Ran et al.; Salom et al.), public health and healthcare (Marei et al.; Silva Campos et al.; Zhu et al.), and health policy and social dynamics (Chaudhury and Banerjee; Stöllberger and Winkler-Dworak; Wagner et al.; Yang et al.) of COVID-19. While certain papers aimed to investigate deeply the mechanisms underlying COVID-19 dynamics, others focused on determining socio-environmental associations useful for predictions and policy discussion. The differentiation between causality and predictability is a rather important point to emphasize due to the unfortunate generalized confusion between the two in "big data" and model-driven investigations that has deep policy implications.

Considering COVID-19 biology, very interestingly, Cueno and Imai found preliminary evidence suggesting that the structural conformation of SARS-CoV-2 (SARS2) spike protein is distinct from other known human-infecting CoVs; this may have triggered the viral pathogenesis (for humans) that mainly relies on the spike glycoprotein (with a furin-like cleavage site (FLC) as a structural feature) located on the virus surface. As a follow up Cueno et al. analyzed the COVID-19 genomic epidemiology network over time and detected nine SARS2 FLC patterns that potentially correspond to nine country clusters associated to the rapid evolution of the SARS2 genome and its infectivity. This evidently shed light into the environmental determinacy of viral biology and infectivity as well as SARS-CoV-2 uniqueness that lead to the pandemic. 


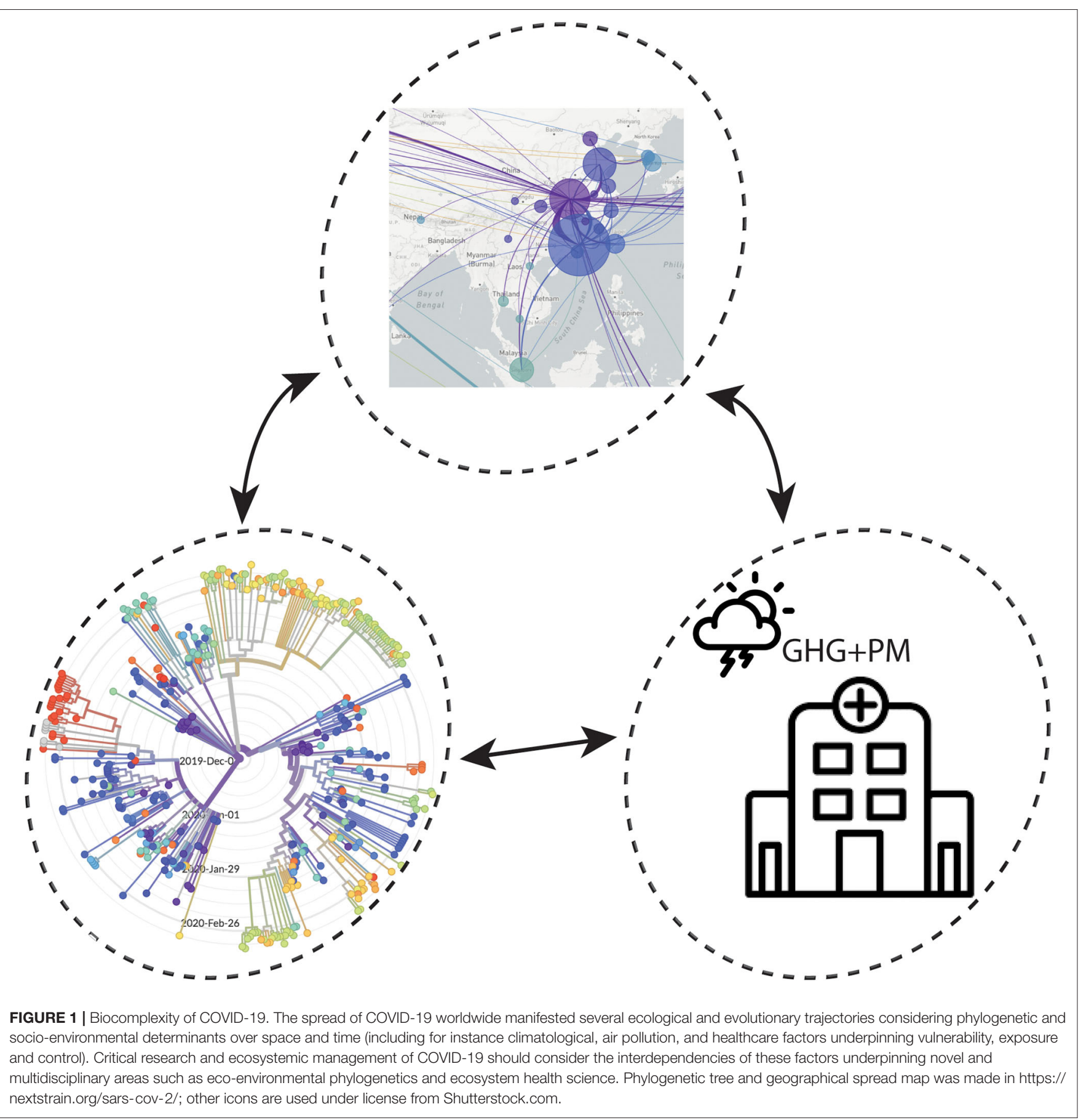

As for COVID-19 environmental vulnerability, Pansini and Fornacca, based on multiscale data from China, found a positive correlation between infections and mortality and Carbon Monoxide (CO), Formaldehyde, PM 2.5, and Nitrogen Dioxide (NO2) (retrieved by Sentinel-5 data) but lack of correlation with population density. These results emphasized how air pollutants are likely population vulnerability factors as suggested by other mechanistic studies highlighting the facilitation of virus spread (including SARS-CoV-2) via pollutant particles. This was also suggested by Salom et al. via detected positive correlations between the inferred reproduction number and pollution levels, as well as with temperature and humidity beyond other health and biological factors (such as cholesterol and blood Rh as individual vulnerabilities).

Contrarily to these findings, Ran et al. found that associations (adjusted by temperature and relative humidity) between three gaseous air pollutants (NO2, SO2, and $\mathrm{CO}$ ) and COVID-19 basic reproductive number (assumed to represent transmissibility) are not statistically correlated. The multivariable linear regression model was used on different pollution estimates from China kriged via meteorological data. 
Overall the discrepancy of findings between these studies emphasizes the caveat of predictions that are always based on input data, their space-time scale, model type and methodology (i.e., how a model is run) as predominant elements. Additionally, a warning is about the lack of consideration of non-linearity in these models that adopt statistical linear correlation for inferring "causality." It is rather important to stress that lack of correlation quite often is a signature of non-linear causation (as demonstrated by evidence on complex systems), and in general absence of correlation does not imply lack of causation between variables; this is easily provable by adopting a probabilistic rather than a categorical deterministic approach.

Yet, based on empirical evidence it is rather clear that persistent air pollution exposure is an ecological multi-scale public health issue where airborne pollutant particulates are likely acting as vector of COVID-19.

As for emphasizing the role of environmental disturbance into the ecology of emerging viruses, Rahalkar and Bahulikar found (based on previously published thesis) that a SARS-like CoV originating from Chinese horseshoe bats (Rhinolophus), was the predicted causative agent of Mojiang mineshaft miners' severe pneumonia-like illness in (2013). This provides very interestingly important clues into the potential ecosystem ontogeny of SARSCoV-2.

As for public health more oriented toward population determinants, with Brazil data Silva Campos et al. tried to identify, via a MCDA model, the underpinning socio-economic and healthcare vulnerability of populations affected by COVID19. Marei et al. emphasized the need of detailed identification of traveler's risk for minimizing COVID-19 spread. Zhu et al. highlighted how China prevention countermeasures for imported cases played an indispensable role in curbing COVID19 spread, and suggested to prolong the 2-week quarantine period for monitoring asymptomatic patients.

In terms of health policy, Yang et al. discussed how COVID19 represented a huge opportunity for clinical research. For this objective Stöllberger and Winkler-Dworak encouraged the sharing of clinical data for defining prognostic indicators and understanding the long-term pulmonary, cardiac, neurologic, and psychiatric consequences of COVID-19.

Lastly, Chaudhury and Banerjee suggested ecotherapy as a form of recovery from psychological effects of COVID- 19 .

In a broader perspective Wagner et al. reviewed economic and behavioral influencing factors of vaccine and antibiotics use and/or refusal globally. This has implications for the acceptance and coverage of COVID-19 vaccine: a topic that is extremely current due to the large vaccination hesitancy in many countries and subpopulations worldwide (also largely associated to misinformation generating risk aversion) that is impacting the curb of COVID-19 spread.

In conclusion, also in light of the diversity of this Special Issue papers, a firm belief should be that research and intervention for this pandemic and complex planetary issues alike, must overcome disciplinary boundaries as well as draw analogies and conclusions from previous events, including small unsuspicious local epidemics. This can raise early warning signals of disastrous systemic outbreaks whose pathogen biology may be largely unknown and extremely transmissible independently of many other factors, such the case of SARS-CoV-2 that "globalized" the world.

We underline the huge role of the environment for the ecology (and biology) of infectious diseases, considering both their emergence and spillover in humans due to environmental transformations, and disease control mediated by inhibitory or enhancing socio-environmental factors such as hygiene and air pollution. Environmental change, due to aggressive local development and ingrained climate change, is dramatically the core of problems like SARS2 emergence where organized and stable ecosystem connections (environment-biota including humans) are compromised. Therefore, in order to study and prevent future pandemics, the hope is to see more natureoriented protection and monitoring before any public health control when the problem already occurred. Additionally, the hope is for science-based solutions in the decision-making process where accurate and salient information is gathered considering objectives, space-time scales, environmental and non-linear dynamics, especially when disease mechanisms are relevant and policy decision are made rather than for mere prediction exercises.

Open transdisciplinary collaborations are certainly creating the optimal platform for this modus operandi, targeting ecosystem or planetary health at the global scale.

\section{AUTHOR CONTRIBUTIONS}

MC developed the original framework of the Special Issue, acted as Chief Editor, and wrote the manuscript and realized the figure. SFP acted as Associate Editor for the Special Issue and revised the writing. All authors contributed to the article and approved the submitted version.

\section{FUNDING}

MC gratefully acknowledges the FY2020 SOUSEI Support Program and Award for Young Researchers awarded by the Executive Office for Research Strategy at Hokkaido University to the Top $20 \%$ scientists in terms of productivity and citations. MC acknowledges the High Talent Scheme ("A" Talents) of the Shenzhen Government as well as the Talents Hiring Program of Tsinghua University, Shenzhen International Graduate School.

Conflict of Interest: The authors declare that the research was conducted in the absence of any commercial or financial relationships that could be construed as a potential conflict of interest.

Publisher's Note: All claims expressed in this article are solely those of the authors and do not necessarily represent those of their affiliated organizations, or those of the publisher, the editors and the reviewers. Any product that may be evaluated in this article, or claim that may be made by its manufacturer, is not guaranteed or endorsed by the publisher.

Copyright $\odot 2021$ Convertino and Pileggi. This is an open-access article distributed under the terms of the Creative Commons Attribution License (CC BY). The use, distribution or reproduction in other forums is permitted, provided the original author(s) and the copyright owner(s) are credited and that the original publication in this journal is cited, in accordance with accepted academic practice. No use, distribution or reproduction is permitted which does not comply with these terms. 